\title{
Analysis of Electromigration in Redundant Vias
}

\author{
Roberto Lacerda de Orio*, Hajdin Ceric*, Sara Carniello ${ }^{\dagger}$, and Siegfried Selberherr* \\ *Institute for Microelectronics, TU Wien, Gußhausstraße 27-29/E360, A-1040 Wien, Austria \\ Email: \{orio|ceric|selberherr\}@iue.tuwien.ac.at \\ †austriamicrosystems AG, Schloss Premstätten, A-8141 Unterpremstätten, Austria \\ Email: sara.carniello@austriamicrosystems.com
}

\begin{abstract}
We analyze the electromigration material transport and stress build-up in dual-damascene interconnect structures with single and double vias. Two cases are studied: in the first, an effective diffusion coefficient is considered, representing the case where grain boundary diffusion is the dominant mechanism and the adhesion between the copper and the capping layer is strong. In the second, the effect of interfaces, based on the assumption that the copper/capping layer interface is the main path for diffusion, is considered. It is shown that the diffusion mechanism has a significant impact on the behavior of the redundant via structure. The influence of the via spacing is also discussed.
\end{abstract}

\section{INTRODUCTION}

Electromigration has been recognized as one of the most important issues for interconnect reliability in modern integrated circuits. In order to reduce its effect, efforts have been spent in developing new integration processes and investigating different materials which produce preferable properties. Such efforts led from originally aluminum interconnects to aluminumcopper alloys, and later to pure copper interconnects fabricated by a dual-damascene technology. The material design encompasses not only the choice of an appropriate interconnect metal, but also the choice of its surrounding materials. Another strategy pursued to improve the electromigration behavior is the introduction of specific geometrical features, such as material reservoirs [1]-[3] and redundant vias [4], [5]. The effect of these geometrical features has been more widely investigated for stress migration than for electromigration. The experiments have shown that stress migration failure is also determined by vacancy transport and mechanical stress buildup.

Ogawa et al. [2] introduced the concept of "active diffusion volume", which is the intersection between the interconnect volume, the diffusion volume, and the stress gradient region, to explain the increased lifetimes observed in structures where the vias were contacted to wide lines. However, Kouno et al. [6] found shorter lifetimes for structures with narrow line extrusions, arguing that the interface between copper, barrier and capping layer acts as a one-dimensional path for vacancy diffusion, which is faster compared to the two-dimensional vacancy flow in a wide plate and, therefore, the active diffusion model does not apply.

Using two or more via contacts between interconnect levels has shown to be a very promising geometrical strategy for preventing stress migration [4], [5] and electromigration [4]. However, the complete understanding, why such geometrical features really enhance interconnect reliability, is still lacking.
The group of Yoshida et al. [5] was the first to closely investigate the effect of redundant vias on stress migration. In their work, no stress migration failure was detected, when two neighboring vias were used. This is because the nucleation of a void underneath the first via relaxes the stress also under the second one, suppressing the driving force for new void formation. Yoshida et al. based their explanation on the concept of active diffusion volume [2].

In this work we present the results of our investigations on the effect of the electromigration stress in interconnect structures with single and double vias. We have used a multiphysics model of electromigration with a complete integration of the mechanical stress with the classical multi-driving force model. Moreover, in our approach we are able to include the effects of the different paths for diffusion, such as material interfaces and grain boundaries, by independently setting the cooresponding diffusion parameters in each specific region. The developed finite element based scheme enables an efficient numerical solution of the three-dimensional formulation of the problem.

\section{Electromigration Modeling}

The transport of vacancies due to the gradient of vacancy concentration, electric field, gradient of temperature, and gradient of mechanical stress, respectively, is [7]

$$
\overrightarrow{J_{v}}=-D_{v}\left(\nabla C_{v}+\frac{Z^{*} e}{k T} C_{v} \nabla \varphi+\frac{f \Omega}{k T} C_{v} \nabla \sigma\right),
$$

where $D_{v}$ is the vacancy diffusion coefficient, $C_{v}$ is the vacancy concentration, $Z^{*} e$ is the effective charge, $Q^{*}$ is the heat of transport, $f$ is the vacancy relaxation ratio, $\Omega$ is the atomic volume, $\sigma$ is the hydrostatic stress, $k$ is Boltzmann's constant, and $T$ is the temperature.

In sites of flux divergence, vacancies will accumulate or vanish, and this vacancy dynamics is described by the continuity equation

$$
\frac{\partial C_{v}}{\partial t}=-\nabla \cdot \vec{J}_{v}+G\left(C_{v}\right)
$$

where $G\left(C_{v}\right)$ is the source function which models vacancy generation and annihilation processes [8].

Sarychev et al. [7] introduced the contribution of the local vacancy dynamics to stress build-up during electromigration, which is given in terms of strain by [9] 


$$
\frac{\partial \varepsilon_{k k}}{\partial t}=\Omega\left[(1-f) \nabla \cdot \vec{J}_{v}+f G\left(C_{v}\right)\right]
$$

where $\varepsilon_{k k}$ is the trace of the strain vector. Such strains are equilibrated by the displacements $\vec{u}=\vec{u}\left(u_{1}, u_{2}, u_{3}\right)$, according to the Lamé-Navier equations

$$
\mu \Delta u_{i}+(\mu+\lambda) \frac{\partial}{\partial x_{i}}(\nabla \cdot \vec{u})=B \frac{\partial \varepsilon_{k k}}{\partial x_{i}}, \quad i=1,2,3
$$

where $\lambda$ and $\mu$ are the Lamé coefficients and $B=\lambda+2 \mu / 3$. Assuming that Hook's Law applies, the stress tensor is

$$
\sigma_{i j}=\sum_{i j k l} \mathbf{C}_{i j k l} \varepsilon_{k l}
$$

where $\mathbf{C}_{i j k l}$ is the compliance matrix and the small displacements approximation is given by

$$
\varepsilon_{i j}=\frac{1}{2}\left(\frac{\partial u_{i}}{\partial x_{j}}+\frac{\partial u_{j}}{\partial x_{i}}\right), \quad i, j=1,2,3 .
$$

\section{Simulation Procedure}

The model presented by the set of equations (1)-(6) is numerically solved by the finite element method implemented in an in-house code for three-dimensional structures. The solving algorithm is depicted in Fig. 1. Each model is handled according to a unique priority list, starting with the electrothermal problem, which is the coupled system of the Laplace and the Fourier equation, respectively,

$$
\begin{aligned}
& \nabla \cdot\left(\gamma_{E} \nabla \varphi\right)=0 \\
& \nabla \cdot\left(\gamma_{T} \nabla T\right)=-p+\rho_{m} c_{p} \frac{\partial T}{\partial t},
\end{aligned}
$$

where $\gamma_{E}$ is the electrical conductivity, $\gamma_{T}$ is the thermal conductivity, $\rho_{m}$ is the mass density, and $c_{p}$ is the specific heat. In equation (8), $p=\gamma_{E}(\nabla \varphi)^{2}$ is the term that accounts for joule heating.

Then, the material transport equations (1) and (2) are solved. Finally, the corresponding stress is determined via Eq. (3)-(6). As shown by Fig. 1, the calculated attributes are transfered from the model of higher priority to the model of lower priority.

\section{Results AND Discussion}

In this section we present our simulation results for interconnect structures with single and double vias for two cases: First, we assume that there is a strong adhesion between the copper and the capping layer, therefore grain boundaries can be considered the main path for diffusion, and we use an effective diffusion in our calculations. For a vacancy bulk diffusion of

$$
D_{b}=D_{0} \exp \left(-\frac{E_{a}}{k T}\right),
$$

with $D_{0}=0.52 \mathrm{~cm}^{2} / \mathrm{s}$ and $E_{a}=1.1 \mathrm{eV}$, we have set an effective diffusion as $D_{\text {eff }}=1000 D_{b}$ [10]. In the second case,

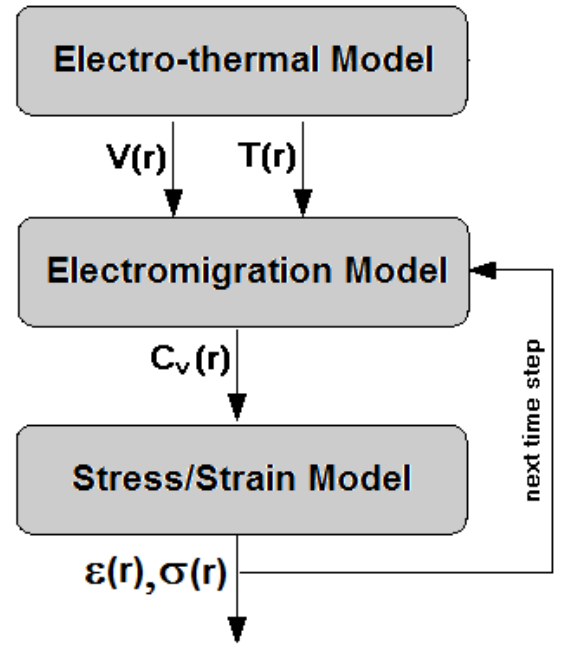

Fig. 1. The complete solving procedure. The electromigration problem and the mechanical problem are solved at each time step.

we consider the copper/capping layer interface as the main diffusion path, where we have set $D_{\text {int }}=100 D_{\text {eff }}$.

The simulations were carried out for an average current density of $8 \mathrm{MA} / \mathrm{cm}^{2}$ and a temperature of $300^{\circ} \mathrm{C}$. We have used the following parameters:

- $\mathrm{Cu}: \mathrm{Z}^{*}=4, \mathrm{f}=0.4, \Omega=1.182 \times 10^{-23} \mathrm{~cm}^{3}, \mathrm{E}=125 \mathrm{GPa}$, $\nu=0.34$

- Ta: $\mathrm{E}=380 \mathrm{GPa}, \nu=0.27$;

- $\mathrm{Si}_{3} \mathrm{~N}_{4}: \mathrm{E}=380 \mathrm{GPa}, \nu=0.27$;

The interconnect structure is shown in Fig. 2. The lower line has a cross section of $0.5 \times 0.2 \mu m^{2}$, the barrier and the capping layer are $25 \mathrm{~nm}$ thick. The thickness of the $\mathrm{SiO}_{2}$ passivation is $500 \mathrm{~nm}$ on the top and bottom of the interconnect.

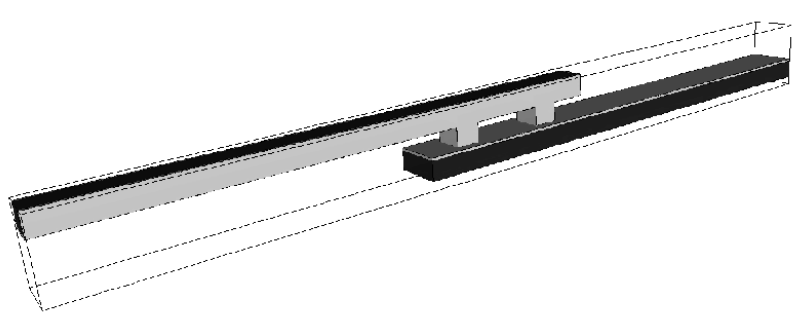

Fig. 2. Dual-damascene interconnect structure.

Fig. 3 shows the hydrostatic stress distribution for the double via structure. The electron flow is from the upper to the lower metal line (downward flow), and therefore it is expected that the vacancies accumulate right under the via region at the 
lower line. As the vacancy has a smaller volume than the metal atom and due to the constraints imposed by the attaching layers and passivation, the accumulation of vacancies in this region leads to the production of local tensile stresses.

The stress build-up for the single via and double via structure for two different distances between the vias is shown in Fig. 4. We have observed that the maximum stress developed for the structure with redundant vias is higher than that for the single via. Such a behavior occurs, because the voltage applied to the ends of the interconnect is kept constant and the addition of the second via reduces the interconnect resistance and increases the electrical current. Consequently, the driving force for material transport along the line is increased, and more vacancies concentrate under the left via of the redundant via structure, producing a higher stress.

Fig. 4 shows that the stress also increases as the distance between the vias becomes larger. It should be pointed out that the maximum vacancy concentration and stress is always located at the bottom of the outer via. However, for the redundant via it is expected that the electric current crowds at the innermost via, where more vacancies should accumulate and produce a higher stress. Thus, our results suggest that the current crowding does not suffice to induce a higher vacancy flux divergence at the inner via.

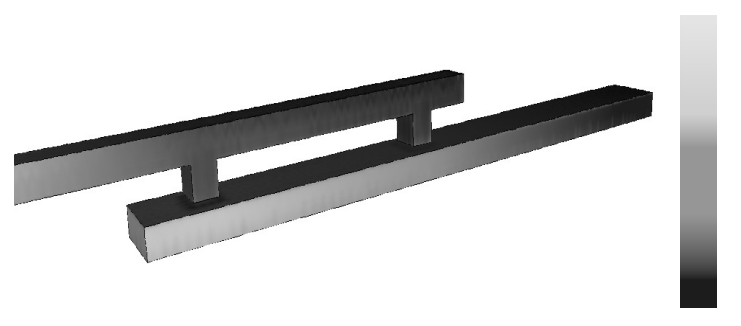

Fig. 3. Detail of the hydrostatic stress distribution in the via region (in $\mathrm{MPa}$ ).

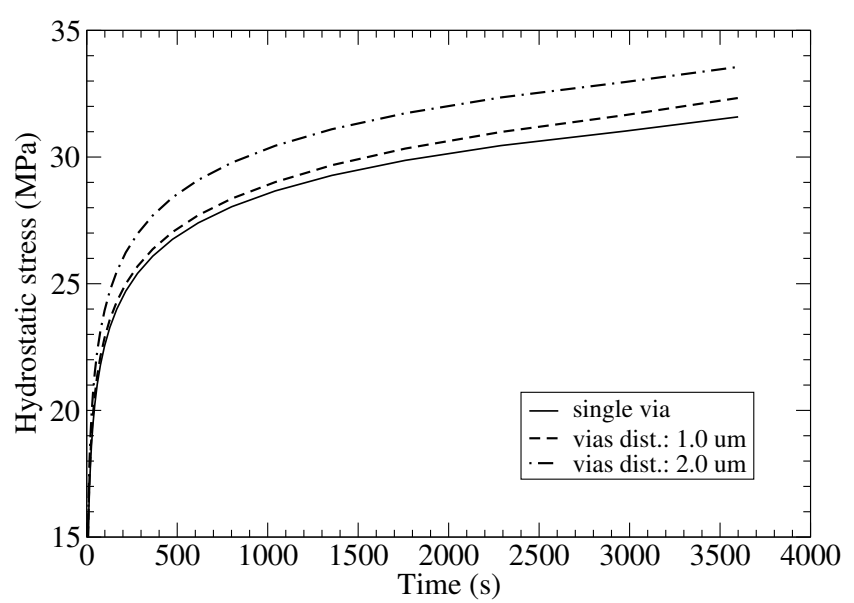

Fig. 4. Maximum hydrostatic stress (tensile) developed under the left via.
Experimental works [11] have shown that the copper/capping layer interface is frequently the main path for material transport in copper dual-damascene interconnects. In order to model this effect, the diffusion coefficient in this interface is increased by a factor 100 in relation to the effective diffusivity of the last section. The corresponding stress buildup is shown in Fig. 5.

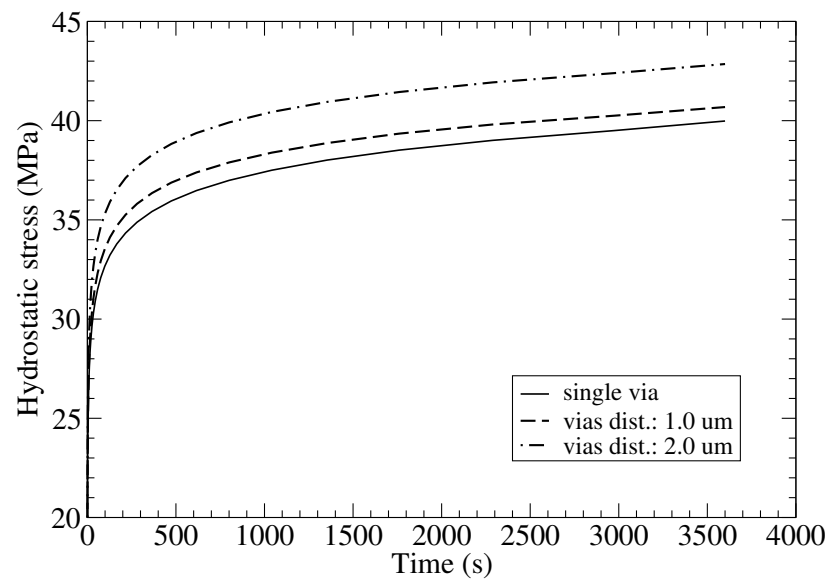

Fig. 5. Maximum hydrostatic stress developed under the left via when the copper/capping layer interface is the main diffusion path.

We can see that the stress values are increased by considering the interfacial diffusion as the dominant mechanism. Moreover, the site where the vacancies concentrate and the maximum stress develops has also changed, similarly to the work of Kouno et al. [6] for stress migration. This is clearly seen in the vacancy concentration and stress distribution in Fig. 6 and Fig. 7, respectively, where we can observe a peak of vacancy concentration and stress also at the border of the inner via.

Two facts are responsible for this behavior: the first one is the current crowding which is expected to occur at this region, as can be seen by the higher current density close to the inner via in Fig. 8. The second one is that the intersection between copper, barrier and capping layer constitutes a site of flux divergence due to a faster diffusion along the copper/capping layer interface in comparison to the copper/barrier layer interface.

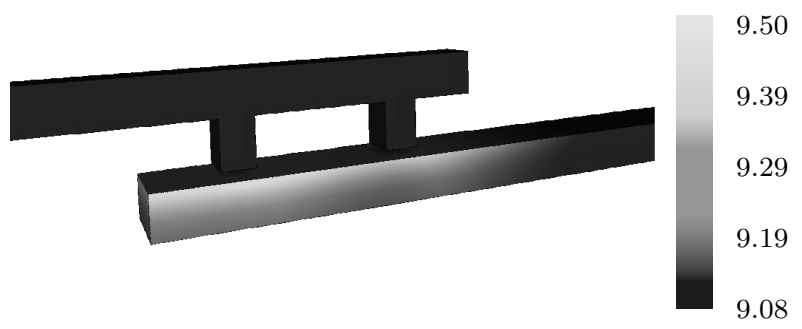

Fig. 6. Vacancy distribution in the double via structure (in $10^{15} \mathrm{~cm}^{-3}$ ). 


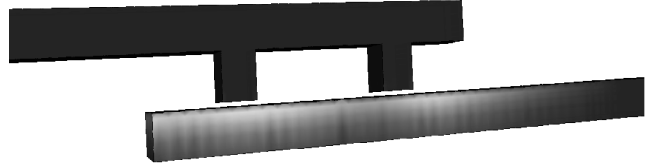

Fig. 7. Early hydrostatic stress distribution under the vias in the double via structure (in $\mathrm{MPa}$ ).

However, over time the vacancy concentration and stress in this region increase, leading to the development of significant gradients. Since these gradients also act as driving forces for material transport, as presented by equation (1), they prevent the continuous accumulation of vacancies driven by the electromigration force and, in addition, help to increase the flux of vacancies through the whole line thickness. This effect is shown in Fig. 9.

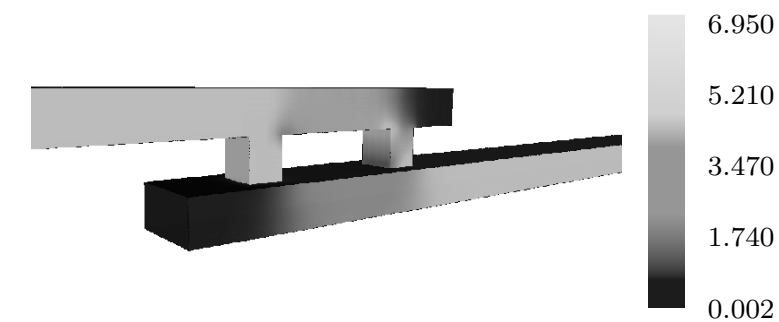

Fig. 8. Current density distribution in the interconnect. The current density is higher next to the inner via, where current crowding increases electromigration transport (in $\mathrm{MA} / \mathrm{cm}^{2}$ ).

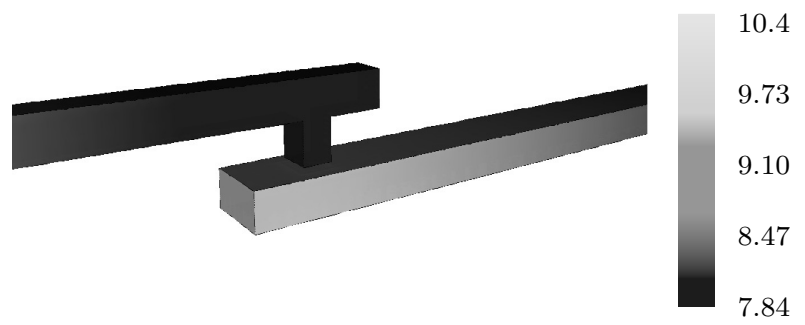

Fig. 9. Vacancy concentration in the single via structure after $1 \mathrm{~h}$ of electromigration stress (in $10^{15} \mathrm{~cm}^{-3}$ ).

\section{CONCLUSION}

The electromigration behavior in interconnect structures with single and double vias was analyzed. In the case of strong adhesion between the copper and the capping layer, an effective diffusion was used to describe the electromigration induced material transport and we observed an increase of the vacancy concentration and stress magnitude at the bottom of the outer via in the redundant via structure, indicating that the current crowding which occurs at the inner via is not sufficient to produce a higher flux divergence at this site.

When diffusion along the copper/capping layer interface is taken into account, vacancies accumulate at the edge of the inner via, at the intersection of copper, barrier and capping layer, and a higher stress develops in this region. Besides the current crowding that occurs at this place, the higher diffusivity along the copper/capping layer interface in comparison to the copper/barrier layer interface helps to accentuate the flux divergence at this site.

We have also observed that significant gradients change the material transport dynamics and vacancies distribute through the whole line thickness, similarly to the effective diffusion case. However, this only occurs for long times, after a peak of stress and vacancy concentration is reached at the copper/capping layer interface, when compared to the bulk values.

\section{ACKNOWLEDGMENT}

Support by the Austrian Science Fund with the project P18825-N14 is gratefully acknowledged.

\section{REFERENCES}

[1] E. T. Ogawa, K. D. Lee, V. A. Blaschke, and P. Ho, "Electromigration Reliability Issues in Dual-Damascene Cu Interconnections," IEEE Trans. on Reliability, vol. 51, no. 4, pp. 403-419, 2002.

[2] E. T. Ogawa, J. W. McPherson, J. A. Rosal, K. J. Dickerson, T. C. Chiu, L. Y. Tsung, M. K. Jain, T. D. Bonifiled, J. C. Ondrusek, and W. R. McKee, "Stress-Induced Voiding under Vias Connected to Wide $\mathrm{Cu}$ Metal Leads," Proc. Intl. Reliability Physics Symp., pp. 312-321, 2002.

[3] H. Matsuyama, M. Shiozu, T. Kouno, T. Suzuki, H. Ehara, S. Otsuka, T. Hosoda, T. Nakamura, Y. Mizushima, M. Miyajima, and K. Shono, "New Degradation Phenomena of Stress-Induced Voiding Inside Via in Copper Interconnects," Proc. Intl. Reliability Physics Symp., pp. 638639, 2007.

[4] A. von Glasow, "Zuverlässigkeitsaspekte von Kupfermetallisierungen in Integrierten Schaltungen," Dissertation, Technische Universität München, 2005.

[5] K. Yoshida, T. Fujimaki, T. Miyamoto, T. Honma, H. Kaneko, H. Nakazawa, and M. Morita, "Stress-Induced Voiding Phenomena for an Actual CMOS LSI Interconnects," Digest Intl. Electron Devices Meeting, pp. 753-756, 2002.

[6] T. Kouno, T. Suzuki, S. Otsuka, T. Hosoda, T. Nakamura, Y. Mizushima, M. Shiozu, H. Matsuyama, K. Shono, H. Watatani, Y. Ohkura, M. Sato, S. Fukuyama, and M. Miyajima, "Stress-Induced Voiding under Vias Connected to "Narrow" Copper Lines," Digest Intl. Electron Devices Meeting, pp. 187-190, 2005.

[7] M. E. Sarychev, Y. V. Zhitnikov, L. Borucki, C. L. Liu, and T. M. Makhviladze, "General Model for Mechanical Stress Evolution During Electromigration,” J. Appl. Phys., vol. 86, no. 6, pp. 3068-3075, 1999.

[8] R. Rosenberg and M. Ohring, "Void Formation and Growth During Electromigration in Thin Films," J. Appl. Phys., vol. 42, no. 13, pp. 5671-5679, 1971.

[9] H. Ceric, R. Heinzl, C. Hollauer, T. Grasser, and S. Selberherr, "Microstructure and Stress Aspects of Electromigration Modeling," StressInduced Phenomena in Metallization, AIP, pp. 262-268, 2006.

[10] V. Sukharev, E. Zschech, and W. D. Nix, "A Model for ElectromigrationInduced Degradation Mechanisms in Dual-Inlaid Copper Interconnects: Effect of Microstructure," J. Appl. Phys., vol. 102, no. 053505, 2007.

[11] A. V. Vairagar, S. G. Mhaisalkar, A. Krishnamoorthy, K. N. Tu, A. M. Gusak, M. A. Meyer, and E. Zschech, "In Situ Observation of Electromigration-Induced Void Migration in Dual-Damascene $\mathrm{Cu}$ Interconnect Structures," Appl. Phys. Lett., vol. 85, no. 13, pp. 25022504, 2004. 